\title{
Flow cytometric assessment of Lactococcus lactis isolates viability after lyophilization
}

\author{
Eliana dos Santos Leandro, , , Graciela Kunrath Lima², Antônio Fernandes de Carvalho ${ }^{3}$, \\ Odilon Gomes Pereira ${ }^{4}$, Célia Alencar de Moraes ${ }^{1}$ \\ ${ }^{1}$ Departamento de Microbiologia, Universidade Federal de Viçosa, Viçosa, Brazil \\ ${ }^{2}$ Departamento de Medicina Veterinária Preventiva, Universidade Federal de Minas Gerais, Belo Horizonte, Brazil \\ ${ }^{3}$ Departamento de Tecnologia de Alimentos, Universidade Federal de Viçosa, Viçosa, Brazil \\ ${ }^{4}$ Departamento de Zootecnia, Universidade Federal de Viçosa, Viçosa, Brazil
}

\section{Email address:}

elianaleandrombi@gmail.com (E.dos S. Leandro)

\section{To site this article:}

Eliana dos Santos Leandro, Graciela Kunrath Lima, Antônio Fernandes de Carvalho, Odilon Gomes Pereira, Célia Alencar de Moraes. Flow Cytometric Assessment of Lactococcus Lactis Isolates Viability after Lyophilization. International Journal of Nutrition and Food Sciences. Vol. 3, No. 5, 2014, pp. 391-396. doi: 10.11648/j.ijnfs.20140305.15

\begin{abstract}
Lactococcus lactis strains tolerance to the lyophilization process is one of the criteria used during the selection of starter cultures in cheese production. The viability of $L$. lactis strains isolated from silage and milk after the lyophilization process was evaluated in the present study by flow cytometry and cell plate counting. The LIVE/DEAD BacLight ${ }^{\mathrm{TM}}$ kit, $^{\mathrm{s}}$ which contains the dyes SYTO 9 and propidium iodide, was used to stain the cells for flow cytometry analysis. The dried cells were also plated on M17 agar and incubated at $30{ }^{\circ} \mathrm{C}$ for 24 hours. The strain L. lactis LBU.1, isolated from buffalo milk, showed the highest tolerance to the lyophilization process, according to flow cytometry and cell plate counting. Although the strains of L. lactis isolated from silage were not the most resistant to the lyophilization process, it is noteworthy that, compared to strains isolated from conventional sources such as cow and goat milk, L. lactis strains from silage showed higher tolerance to the lyophilization process.
\end{abstract}

Keywords: Lactococcus Lactis, Lyophilization, Viability, Flow Cytometry

\section{Introduction}

Lactococcus lactis is a lactic acid bacterium of great importance for the dairy industry as a starter culture in cheese production. Strains of L. lactis selected for cheese production must have high acidification activity. Lyophilization is used as a method for preserving lactic acid bacteria cultures. However, the stress generated during the process of lyophilization affects the physiological state of the cells, leading to loss of viability and acidification activity [1]. Some studies have suggested the need to maintain a high level of viability and acidification activity of lactic acid bacteria during freezing, lyophilization and prolonged storage $[2,3]$. Therefore, studies have been carried out to improve the resistance of lactic acid bacteria to the lyophilization process. The use of protective substances [4] and the application of sub-lethal stress [5] before the lyophilization process have been employed to increase the tolerance of cells during lyophilization or freezing. However, in addition to these treatments, it is necessary that the strains used have a natural resistance to the lyophilization process, independent of the treatments that are applied prior to lyophilization.

The viability of the starter cultures is traditionally quantified by the cell plate count method, which evaluates the ability of cells to grow in an appropriate medium, and hence the population of cultivable bacteria is determined. Colony plaque counting is the conventional method to quantify survival; however, due to the low sensitivity of this technique, the selection of $L$. lactis strains with increased tolerance to lyophilization could be hampered. Thus, more sensitive and rapid methods that provide information about cellular integrity are needed to monitor survival [6].

Flow cytometry is a rapid method to analyze individual cells. It uses light scattering criteria and specific fluorescent dyes to obtain information about the structural and functional characteristics of the cell, respectively [7]. The subpopulations may be distinguished based on different 
fluorescence characteristics or by light scattering, which is related to cell volume and structure and surface properties. Many fluorescent dyes have been used to analyze diverse physiological parameters, such as membrane integrity [8], activity of intracellular enzymes [9], cell vitality [10], membrane potential [11] and intracellular $\mathrm{pH}$ [12]. The LIVE/DEAD kit (BacLight ${ }^{\mathrm{TM}}$, Invitrogen), which measures cell viability, has been used in combination with flow cytometry to estimate the number of viable bacteria and also the total count of bacteria [13]. The BacLight ${ }^{\mathrm{TM}}$ kit is composed of two dyes that bind to nucleic acids: SYTO 9, which stains all cells with green fluorescence, and propidium iodide (PI), which only enters cells with compromised membrane integrity, staining the cells with red fluorescence [14].

Strains of L. lactis isolated from plant material produce different compounds associated with flavor, what is not observed with the strains isolated from milk or cheese. In addition, these strains have shown a greater tolerance to osmotic stress conditions caused by $\mathrm{NaCl}$ and higher tolerance to acidic environments [15]. However, their resistance to the lyophilization process has not been studied. Therefore, the aim of the present study was to evaluate the lyophilization tolerance of $L$. lactis strains isolated from silage and milk by flow cytometry. In addition, we also used the cell plate count method to evaluate the efficiency of the two methods in differentiating strains resistant and susceptible to lyophilization.

\section{Materials and Methods}

\subsection{Strains and Storage Conditions}

Five strains isolated from different sources were studied: L. lactis LVA. 2 (cow milk), L. lactis LBU.1 (buffalo milk), L. lactis LCA .5 (goat milk), L. lactis SBR .5 (grass silage) and L. lactis SAM. 12 (forage peanut silage). The strains were grown in M17 broth supplemented with $0.5 \%$ glucose for 14 hours at $30{ }^{\circ} \mathrm{C}$, and a $1 \%$ inoculum of this culture was added to $10 \mathrm{~mL}$ of the same culture medium and incubated for 7 hours at $30{ }^{\circ} \mathrm{C}$. Aliquots of $1 \mathrm{~mL}$ of culture collected at the early stationary growth phase were centrifuged $(10,000$ $\times \mathrm{g}, 5 \mathrm{~min}$ at $4{ }^{\circ} \mathrm{C}$ ). The cell pellet was then washed twice with $0.1 \%(\mathrm{w} / \mathrm{v})$ peptone water and centrifuged again. The pellet was resuspended in $1 \mathrm{~mL}$ of peptone water and stored at $-80{ }^{\circ} \mathrm{C}$ for 3 hours. Frozen samples were dried in a lyophilizer (Enterprise II, Terroni ${ }^{\circledR}$ ) for 7 hours. Lyophilized samples were reconstituted in $1 \mathrm{~mL}$ of $0.1 \%$ peptone water for 15 minutes at room temperature. After reconstitution, the samples were analyzed by flow cytometry and cell plate counting.

\subsection{Analysis of Cell Integrity}

\subsubsection{Preparation of Control Samples}

The active culture of each strain inoculated ( $1 \%$ inoculum) into M17 broth was incubated for 5 hours at $30{ }^{\circ} \mathrm{C}$ to collect fresh cells in the exponential growth phase, which were used as a positive control. Aliquots of $1 \mathrm{~mL}$ from these cells were incubated at $70{ }^{\circ} \mathrm{C}$ for 10 minutes to obtain dead cells, which were used as a negative control. Different proportions of live and dead cells were mixed to assess the ability of the dyes to discriminate between live and dead cells.

\subsubsection{Fluorescent Dyes and Staining Procedure}

The fluorescent dyes SYTO 9 and PI of the LIVE/DEAD ${ }^{\circledR}$ BacLight $^{\mathrm{TM}}$ bacterial viability kit (Molecular Probes, Invitrogen) were used. The staining protocol provided by the manufacturer had some modifications. Aliquots of the stock solutions of SYTO $9(3.34 \mathrm{mM})$ and PI $(20 \mathrm{mM})$ were diluted in a $0.85 \%(\mathrm{w} / \mathrm{v})$ saline solution, to give concentrations of SYTO 9 and PI of $0.83 \mathrm{mM}$ and $5 \mathrm{mM}$, respectively. Dehydrated cells reconstituted in $0.1 \%$ peptone water were diluted in $0.85 \%(\mathrm{w} / \mathrm{v})$ saline solution to obtain $10^{4}-10^{5} \mathrm{CFU} \cdot \mathrm{mL}^{-1}$. Aliquots of $100 \mu \mathrm{L}$ of this diluted cell suspension were added to $900 \mu \mathrm{L}$ of $0.85 \%$ saline solution, and $1.5 \mu \mathrm{L}$ of each dye was added. The samples were incubated for 15 minutes at room temperature in the dark and then centrifuged $\left(13,000 \times g, 3\right.$ minutes at $\left.4{ }^{\circ} \mathrm{C}\right)$. The pellet was resuspended in $300 \mu \mathrm{L}$ of $0.85 \%(\mathrm{w} / \mathrm{v})$ saline solution before flow cytometry analysis.

\subsubsection{Analysis by Flow Cytometry}

Flow cytometry analyses were performed with a Guava EasyCyte Plus ${ }^{\circledR}$ cytometer. The cytometer was equipped with an argon laser that emitted blue light at $488 \mathrm{~nm}$. The red fluorescence of cells stained with PI and the green fluorescence of cells stained with SYTO 9 were detected by the $583 / 26 \mathrm{~nm}$ and $525 / 30 \mathrm{~nm}$ filters, respectively. The analysis was performed using a logarithmic scale, the voltages of the detectors were adjusted using unstained cells, and compensations were performed using cells labeled with a single dye. The gates in the FSC/SSC graphs were used to discriminate bacteria. Analyses were performed using low flow $\left(0.24 \mu \mathrm{l} \mathrm{sec}{ }^{-1}\right)$, and event data $(10,000$ events per sample, counted in the FSCxSSC bacteria gate) were collected and analyzed using Cytosoft 5.3 Guava ${ }^{\circledR}$.

\subsubsection{Data Analysis}

Data were collected and analyzed using the software Cytosoft 5.3 Guava ${ }^{\circledR}$. The subpopulations were identified using dot plots. The gates were set in the dot plots of FSC versus SSC and green fluorescence versus red fluorescence. Data were analyzed using statistical tables that indicated the number and percentage of stained cells determined by each detector and the fluorescence intensity of each signal in each defined gate or region of interest.

\subsection{Cell plate Counting}

The cultivability of the lyophilized cells was evaluated by plate count method. After several serial dilutions in $0.85 \%$ saline solution, selected dilutions were plated on M17 agar according to the micro-drop technique [16] and incubated at $30{ }^{\circ} \mathrm{C}$ for 24 hours. Results are from three replicates. 


\section{Results and Discussion}

\subsection{Analysis of Lyophilized Strains by Flow Cytometry}

\subsubsection{Analysis of the Positive and Negative Controls}

The staining of fresh (Figure 1a) and heat-killed $\left(70^{\circ} \mathrm{C}\right.$ for 10 minutes) cells (Figure 1b) with SYTO 9 was evaluated.

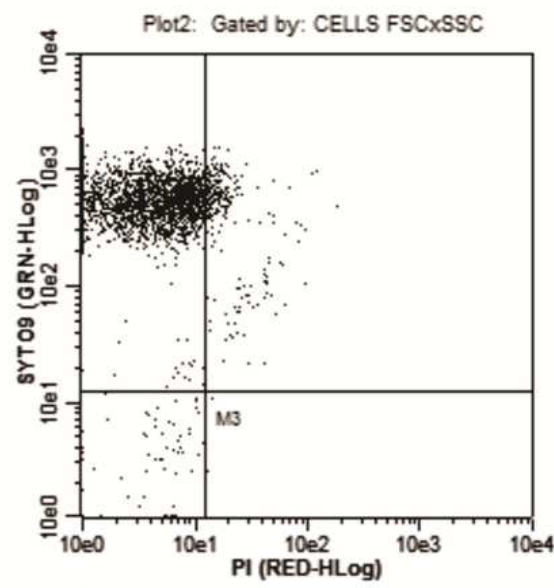

(a)

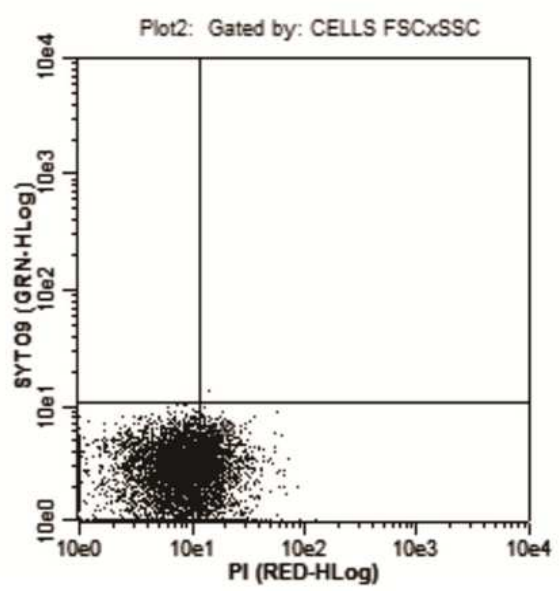

(c)

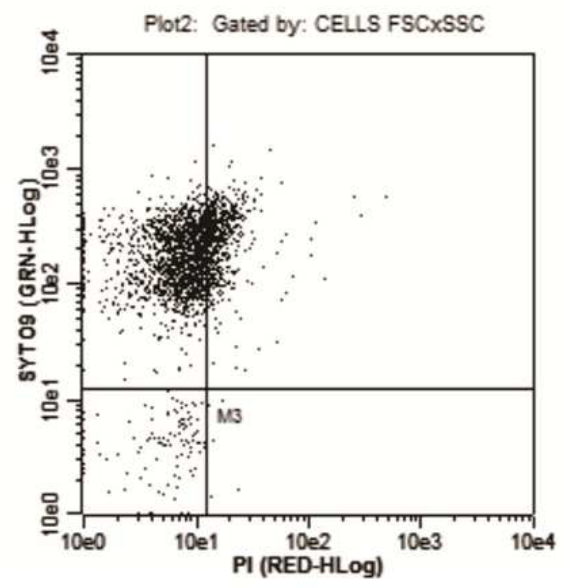

(b)

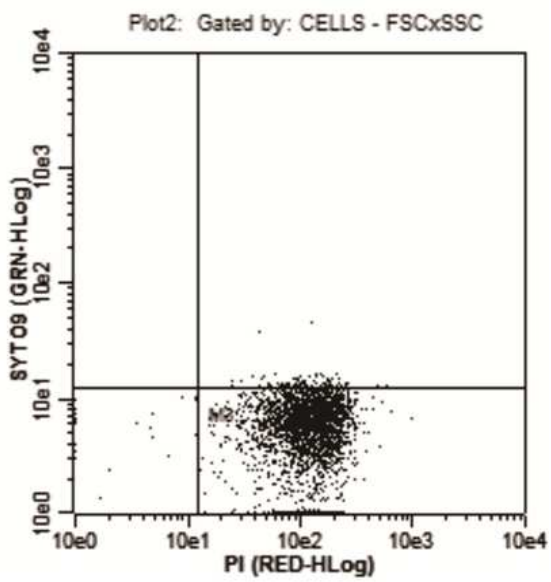

(d)

Figure 1. Dot plot representing the SYTO 9 and PI fluorescence signals of L. lactis LVA. 2. Fresh cells (a) and heat-killed cells (b) stained with SYTO 9. Fresh cell (c) and heat-killed cell (d) stained with PI.

Green fluorescence was observed in both fresh and heated-killed cells (Figure 1a and 1b). However, green fluorescence was more pronounced in dead cells (Figure 1b). Dye penetration was possibly facilitated in dead cells due to membrane damage, resulting in higher green fluorescence intensity. On the other hand, parts of the fresh cells were stained with PI, (Figure 1c). We expected unstained cells only, but even with the cells being collected during exponential growth, the products of the cell metabolism (lactic acid, $\mathrm{H}_{2} \mathrm{O}_{2}$, diacetyl, bacteriocins) can compromise cells integrity. A similar result was observed when the fresh cell suspension was stained simultaneously with SYTO 9 and PI (Figure 2a). Dead cells were properly stained with PI (Figure 1d).
Dead cells stained with the two dyes had most of the population stained with green and red fluorescence, which is a satisfactory result (Figure 2b). The detection of the proportions of fresh $(50 \%)$ and heat-killed cells $(50 \%)$ in the same mixture, simultaneously labeled with SYTO 9 and PI, confirmed the discrimination ability of the dyes in the quantification of live and dead cells (Figure 2c). Although the proportion of dead cells was greater than $50 \%$, we believe that cells that suffered some sort of damage during culture may have been included in this total. Despite the interference of the complexity of the physiological state of the cells during culture, discrimination between live and dead cells in control samples was satisfactory. 
(a)

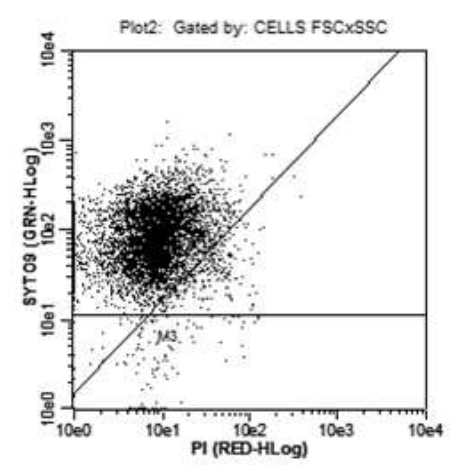

(b)

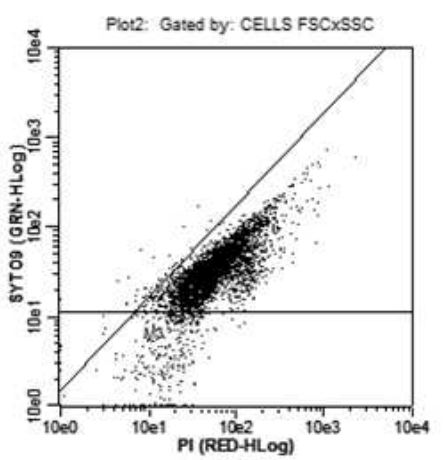

(c)

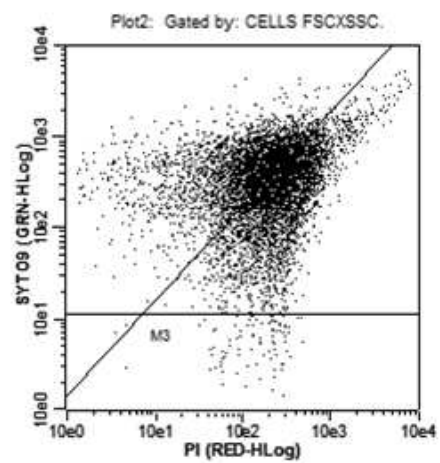

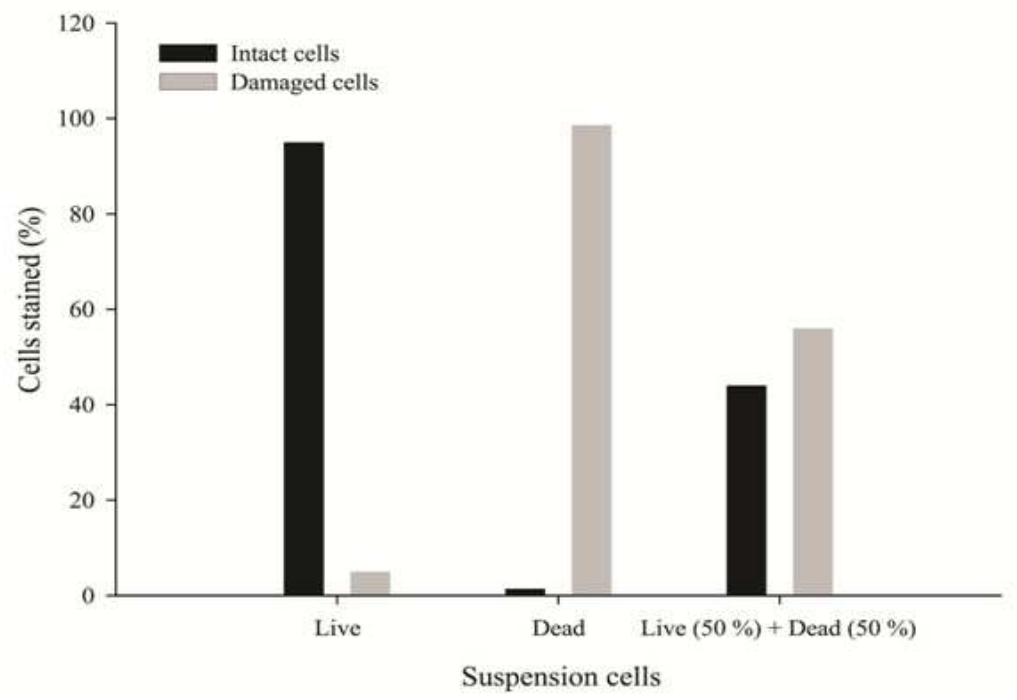

Figure 2. Multiparameter plot obtained after double staining (SYTO 9/PI) of fresh living cells (a), fresh dead cells (b) and the mixture of L. lactis LVA. 2 heat-killed cells $(50 \%)$ and fresh cells $(50 \%)$ (c)

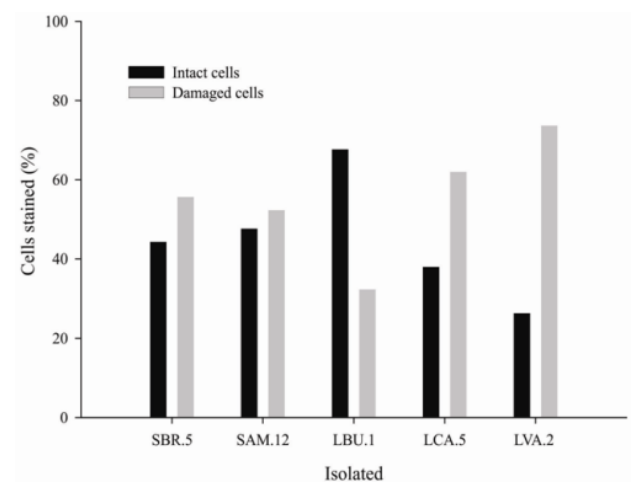

Figure 3. Percentages of viable and damaged cells of the five strains of $L$. lactis after the lyophilization process, determined by flow cytometry. Data are the mean values from three replicates.

\subsubsection{Determination of Viable and Damaged Cells of $L$. Lactis Strains After Lyophilization}

The PI and SYTO 9 dyes were used simultaneously to assess the viability of five strains of $L$. lactis isolated from different sources after the lyophilization process. Figure 3 shows the percentage of cells stained with SYTO 9 (intact cells) and with PI (damaged cells).

Visible differences were observed among the five strains. The LBU. 1 strain showed the highest percentage of intact cells, and the LVA. 2 strain had the lowest percentage of intact cells. Cell suspensions were lyophilized without the addition of protective substances (sugars, amino acids, polysaccharides and complex substances). The absence of these substances during lyophilization may have facilitated the identification of strains resistant and susceptible to lyophilization. Furthermore, because PI does not necessarily stain only dead cells, but also cells with slightly damaged plasma membranes, this ultimately makes the flow cytometry technique very sensitive and thus facilitates the identification of strains resistant and susceptible to lyophilization [17].

Strains SBR. 5 and SAM. 12, isolated from grass and forage peanut silage, respectively, were not the most tolerant strains to the lyophilization process. It is noteworthy that the LBU. 1 strain, isolated from buffalo milk, and with increased tolerance to the lyophilization process, is not a 
conventional source for the isolation of L. lactis strains. However, the lyophilization tolerance of the strains isolated from silage was higher only when compared with the strains LVA. 2 and LCA. 5, which were isolated from conventional ecosystems such as cow and goat milk, respectively. It is possible that the strains isolated from silage have undergone selection for resistance mechanisms under the conditions of their host plant physiology, which may have contributed to the lyophilization tolerance.

Overall, the percentage of viable cells detected by flow cytometry was low. This was most likely due to the absence of cryoprotective substances and the collection of the cells in the early stationary phase of growth. Usually, cells collected at the end of the stationary phase have activated various resistance mechanisms due to nutritional stress, leading to an increased tolerance to other stress conditions, such as lyophilization [18]. Related bacteria species show high percentages of viable cells, as seen with the strains Lactobacillus acidophilus LA-5 and Bifidobacterium animalis subsp. lactis BB-12 after being collected in the late stationary phase and lyophilized in a suspension containing $10 \%$ sucrose and $10 \%$ glucose [13]. In addition to these factors, the conditions for culture reconstitution after the dehydration process, such as the type of cell medium and the reconstitution time long, are major factors in the recovery of cell integrity [7]. These factors may have compromised our ability to obtain a higher percentage of viable cells. Moreover, small lesions can be detected by flow cytometry, but these may not necessarily impair cell viability.

\subsection{Analysis by Plate Count}

The results obtained by cell plate count method (Figure 4) showed no direct relationship with the flow cytometry results.

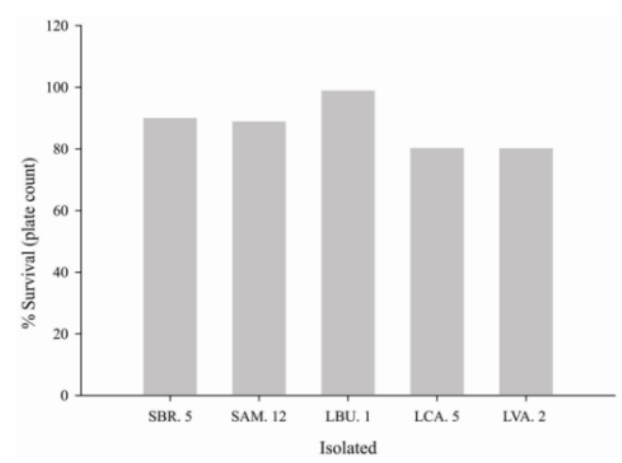

Figure 4. Survival percentages of the L. lactis strains after the lyophilization process, determined by the plate count method. The results are the mean values of three replicates.

A high percentage of damaged cells were detected by flow cytometry, even though a large population of cells was shown to be cultivable by plate count. A fraction of cells stained with PI in a population of damaged cells detected by flow cytometry are able to grow on agar plates [19]. This occurs because PI is a sensitive dye that stains damaged live cells and thus is a poor label for dead cells [17]. Most of the cells stained with PI likely had reversible damage and were able to recover and grow in optimal culture conditions. Some studies have demonstrated, for many microorganisms, a high correlation between the plate count and flow cytometry methods $[20,21,22]$, while others have shown that flow cytometry analysis is not an indicator of cultivability $[23,24]$. These variations may be related to differences in the species and strains tested, as well as in the dyes and equipment used for analysis. In the present study, although a relationship between the two techniques was not observed, the similarities and differences in survival among strains were essentially maintained between cell plate counting and flow cytometry. The survival percentages of the five $L$. lactis strains obtained by the plate count method were similar to each other, what makes the selection of strains resistant and susceptible to the lyophilization process difficult.

\section{Conclusion}

Flow cytometry with the SYTO 9 and PI dyes is a sensitive method for the identification of L. lactis strains susceptible and resistant to lyophilization. The lyophilization tolerance observed for the L. lactis strains LBU. 1, SBR. 5 and SAM. 12 might be related to the characteristics of the environment in which they were isolated. Moreover, there is an opportunity for starter culture companies to incorporate this technique into the culture production process to improve their operating conditions.

\section{Acknowledgements}

The authors thank the Conselho de Desenvolvimento Científico e Tecnológico (CNPq), Brasília, Brazil and the Coordenação de Aperfeiçoamento de Pessoal de nível Superior (CAPES), Brasília, Brazil, for providing financial support for this research.

\section{References}

[1] Coulibaly, I., Dubois-Dauphin, R., Destain, J., Fauconnier, M., Lognay, G., \& Thonart, P. (2010). The resistance to freeze-drying and to storage was determined as the cellular ability to recover its survival rate and acidification activity. International Journal of Microbiology 1-9.

[2] Selwal, K., Selwal, M., \& Gandhi, D. N.(2011). Effect of freeze drying process on some properties of Streptococcus thermophilus. Brazilian Journal of Microbiology 42: 1500-1505.

[3] Strasser, S., Neureiter, M., Geppl, M., Braun, R., \& Danner, H.(2009). Influence of lyophilization, fluidized bed drying, addition of protectants, and storage on the viability of lactic acid bacteria. Journal of Applied Microbiology 107: 167-177.

[4] Pyar, H., \& Peh, K.(2011). Effect cryoprotective agents on survival and stability of Lactobacillus acidophilus cultured in food-grade medium. International Journal of Dairy Technology 64: 578-584. 
[5] Simões, C., Alakomi, H., Maukonen, J., \& Saarela, M.(2010) Expression of clpL1 and clpL2 genes in Lactobacillus rhamnosus VTT E-97800 after exposure to acid and heat stress treatments or to freeze-drying. Beneficial Microbes 1: 253-257.

[6] Bouix, M., \& Ghorbal, S. (2012). Rapid enumeration of Oenococcus oeni during malolactic fermentation by flow cytometry. Journal of Applied Microbiology 1-7.

[7] Muller, J. A. Stanton, C., Sybesma, W., Fitzgerald, G. F., \& Ross, R. P.(2010). Reconstitution conditions for dried probiotic powders represent a critical step in determining cell viability. Journal of Applied Microbiology 108: 1369-1379.

[8] Soejima, T., Minami, J., \& Iwatsuki, K.(2012). The exclusive use of flow cytometry to evaluate the antibiotic-susceptibility. Biochimica et Biophysica Acta 1820: 1980-1986.

[9] Paparella, A., Taccogna, L., Aguzzi, I., Chaves-López, C., Serio, A., Marsilio, F., \& Suzzi, G.(2008). Flow cytometric assessment of the antimicrobial activity of essential oils against Listeria monocytogenes. Food Control 19: 1174-1182.

[10] Michal, K., Baszczynski, M., Lehnert, R., Mota, A., Teixeira, J. A., \& Brányik, T. (2009). Flow cytometry for age assessment of a yeast population and its application in beer fermentations. Journal of the Institute of Brewing 115: 253-258.

[11] Xu, J., Zhou, F., Ji, B. P., Pei, R. S., \& Xu, N.(2008). The antibacterial mechanism of carvacrol and thymol against Escherichia coli. Letters in Applied Microbiology 47: 174-179.

[12] Marchetti, A., Lelong, E., \& Cosson, P. (2009).A measure of endosomal pH by flow cytometry in Dictyostelium. BMC Research Notes 2-7.

[13] Kramer, M., Obermajer, N., Matijasic, B. B., Rogelj, I., \& Kmetec, V. (2009). Quantification of live and dead probiotic bacteria in lyophilized product by real-time PCR and by flow cytometry. Applied Microbiology Biotechnology $84: 1137-1147$.

[14] Boulos, L., Prévost, M., Barbeau, B., Coallier, J., \& Desjardins, R. (1999). Live/Dead ${ }^{\mathbb{R}}$ BacLight $^{\mathrm{TM}}$ : application of a new rapid staining method for direct enumeration of viable and total bacteria in drinking water. Journal Microbiology Methods 37: 77-86.
[15] Nomura, M., Kobayashi, M., Narita, T., Kimoto-Nira, H., \& Okamoto, T.(2006). Phenotypic and molecular characterization of Lactococcus lactis from milk and plants. Journal of Applied Microbiology 101: 396-405.

[16] Morton, R. D. Aerobic plate count. In F. P. Downes, K. Ito (Eds.),(2001). Compendium of methods for the microbiological examination of foods ( $4^{\text {th }}$ ed.), (pp. 1183-1193). Washington D.C.: American Public Health Association.

[17] Novo, D. J., Perlmutter, N. G., Hunt, R. H., \& Shapiro, H. M.(2000). Multiparameter flow cytometric analysis of antibiotic effects on membrane potential, membrane permeability, and bacterial counts of Staphylococcus aureus and Micrococcus luteus. Antimicrobial Agents and Chemotherapy 44: 827-834.

[18] Morgan, C. A., Herman, N., White, P. A., \& Vesey, G.(2006). Preservation of micro-organisms by drying: A review. Journal of Microbiology Methods 66:183-193

[19] Papadimitriou, K., Pratsinis, H., Nebe-von-Caron, G., Kletsas, D., \& Tsakalidou, E.(2006). Rapid assessment of the physiological status of Streptococcus macedonicus by flow cytometry and fluorescence probes. International Journal of Food Microbiology 111: 197-205.

[20] Bouix, M., \& Leveau, J. Y. (2001). Rapid assessment of yeast viability and yeast vitality during alcoholic fermentation . Journal of the Institute of Brewing 107: 217-225.

[21] Bunthof, C. J. \& Abee, T. (2002). Development of a flow cytometric method to analyze subpopulations of bacteria in probiotic products and dairy starters. Applied and Environmental Microbiology 68: 2934-2942.

[22] Rault, A., Béal, C., Ghorbal, S., Ogier, J., \& Bouix, M. (2007) Multiparametric flow cytometry allows rapid assessment and comparison of lactic acid bacteria viability after freeze and during frozen storage. Cryobiology 55: 35-43.

[23] Amor, K. B., Breeuwer, P., Verbaarschot, P., Rombouts, F. M., Akkermans, A. D., Vos, W. M., \& Abee, T. (2002). Multiparametric flow cytometry and cell sorting for the assessment of viable, injured, and dead Bifidobacterium cells during Bile Salt Stress. Applied and Environmental Microbiology 68: 5209-5216.

[24] Bunthof, C. J., Braak, V., Breeuwer, P. Rombouts, F. M., \& Abee, T. (1999). Rapid fluorescence assessment of the viability of stressed Lactococcus lactis. Applied and Environmental Microbiology 65: 3681-3689. 